\title{
Erratum to: Foundations of the Quantum Chromodynamics
}

\section{Erratum to:}

Chapter 2 in: J. Wang, QCD Higher-Order Effects and Search for New Physics, Springer Theses, DOI 10.1007/978-3-662-48673-3_2

The original version of the book was inadvertently published with an incorrect Eq. 2.29 in chapter 2. The chapter and the book have been updated with the change.

The updated original online version for this chapter can be found 10.1007/978-3-662-48673-3_2

\footnotetext{
J. Wang $(\bowtie)$

Johannes Gutenberg University, Mainz, Germany

e-mail: jian.wang@uni-mainz.de 